\title{
Which Is Reactive in Alkaline Solution, Boronate Ion or Boronic Acid ? - Kinetic Evidence for Reactive Trigonal Boronic Acid in Alkaline Solution -
}

\author{
Satoshi Iwatsuki, Shihoko Nakajima, Masahiko Inamo, Hideo D. Takagi and Koji Ishihara
}

\section{Experimental details}

2,4-Difluorophenylboronic acid (2,4- $\mathrm{F}_{2} \mathrm{PhB}(\mathrm{OH})_{2}$, Aldrich), 3-trifluoromethylphenyl-boronic acid (3- $\mathrm{CF}_{3} \mathrm{PhB}(\mathrm{OH})_{2}$, Tokyo Chemical Industry Co. Ltd. (TCI)), and phenylboronic acid $\left(\mathrm{PhB}(\mathrm{OH})_{2}\right.$, Aldrich) were recrystallized once from water. 2,2'-Biphenol (TCI) was recrystallized from toluene. 2,3-Dihydroxynaphthalene (Aldrich) was recrystallized once from water. Perchloric acid (60\% UGR for trace analysis) and $5 \mathrm{M}$ sodium hydroxide solution (5 $N$ ) (Kanto Chemical Co. Inc.) were used without further purification. A solution of sodium perchlorate was prepared as described previously [1]. Twice distilled water was used for preparation of all solutions. The ionic strength was kept with sodium perchlorate. Spectrophotometric measurements were performed with a rapid-scan/stopped-flow spectrophotometer USP600 (UNISOKU Scientific Instruments). The pH measurements were performed with a pH meter HM-30G (TOA-DKK).

Kinetic measurements were carried out at $I=0.10 \mathrm{M}$ and $T=25.0 \pm 0.1^{\circ} \mathrm{C}$. Rate constants for all of the complex formations of the boronic acids were measured by following the absorbance change (the decrease at $308 \mathrm{~nm}$ for the 2,2'-biphenol systems and the increase at $328 \mathrm{~nm}$ for the 2,3-dihydroxynaphthalene system) with time under the pseudo first-order conditions that the total concentration of boronic acid $\left(C_{\mathrm{B}}\right)$ was in large excess over the ligand $\left(C_{\mathrm{L}}\right)$. All the reaction curves were excellently single exponential within at least 10 half-lives, for which conditional pseudo first-order rate constants, $k_{\mathrm{obs}}$, were determined by applying a least-squares fitting. The pH values were determined after completion of the reaction.

The rate constants in eq 3 in the text were determined by applying a non-linear least-squares fitting to $k_{\mathrm{obs}}$ vs. $C_{\mathrm{B}}$ plots (Figures 1 , S1, and S3 for the reactions of $\mathrm{RB}(\mathrm{OH})_{2}$ with 2,2'-biphenol, and Figure 3 for the reaction with 2,4- $\mathrm{F}_{2} \mathrm{PhB}(\mathrm{OH})_{2}$ with 2,3-dihydroxynaphthalene), and the results were tabulated in Table S1.

Ref. [1] S. Funahashi, K. Haraguchi and M. Tanaka, Inorg. Chem., 1977, 16, 1349. 
Table S1. The rate constants obtained from non-linear least-squares analyses of $k_{\mathrm{obs}}$ vs. $C_{\mathrm{B}}$ plots (Figure 1, S1, S3 for the 2,2'-biphenol reaction systems, and Figure 3 for the 2,3-dihydroxynaphthalene system) ${ }^{a}$

\begin{tabular}{|c|c|c|c|}
\hline reaction system & rate constant & & \\
\hline \multirow{4}{*}{ 2,4- $\mathrm{F}_{2} \mathrm{PhB}(\mathrm{OH})_{2}+2,2^{\prime}$-biphenol } & $k_{1}=$ & $(1.11 \pm 0.04) \times 10^{3}$ & $\mathrm{M}^{-1} \mathrm{~s}^{-1}$ \\
\hline & $k_{2} K_{\mathrm{a}}^{\mathrm{L}}+k_{3} K_{\mathrm{a}}^{\mathrm{B}}=$ & $(1.21 \pm 0.09) \times 10^{-5}$ & $\mathrm{~s}^{-1}$ \\
\hline & $k_{-1}=$ & $(1.04 \pm 0.05) \times 10^{7}$ & $\mathrm{M}^{-1} \mathrm{~s}^{-1}$ \\
\hline & $k_{-2}+k_{-3}$ & $-b$ & \\
\hline \multirow[t]{4}{*}{ 3- $\mathrm{CF}_{3} \mathrm{PhB}(\mathrm{OH})_{2}+2,2$ '-biphenol } & $k_{1}=$ & $(5.91 \pm 0.22) \times 10^{2}$ & $\mathrm{M}^{-1} \mathrm{~s}^{-1}$ \\
\hline & $k_{2} K_{\mathrm{a}}^{\mathrm{L}}+k_{3} K_{\mathrm{a}}^{\mathrm{B}}=$ & $(5.25 \pm 0.28) \times 10^{-6}$ & $\mathrm{~s}^{-1}$ \\
\hline & $k_{-1}=$ & $(5.03 \pm 0.89) \times 10^{6}$ & $\mathrm{M}^{-1} \mathrm{~s}^{-1}$ \\
\hline & $k_{2}+k_{-3}=$ & $(3.10 \pm 1.10) \times 10^{-2}$ & $\mathrm{~s}^{-1}$ \\
\hline \multirow[t]{4}{*}{$\mathrm{PhB}(\mathrm{OH})_{2}+$ 2,2'-biphenol } & $k_{1}=$ & $(1.31 \pm 0.06) \times 10^{2}$ & $\mathrm{M}^{-1} \mathrm{~s}^{-1}$ \\
\hline & $k_{2} K_{\mathrm{a}}^{\mathrm{L}}+k_{3} K_{\mathrm{a}}^{\mathrm{B}}=$ & $(1.61 \pm 0.04) \times 10^{-6}$ & $\mathrm{~s}^{-1}$ \\
\hline & $k_{-1}$ & $-b$ & \\
\hline & $k_{-2}+k_{-3}=$ & $0.253 \pm 0.003$ & $\mathrm{~s}^{-1}$ \\
\hline \multirow[t]{4}{*}{ 2,4- $\mathrm{F}_{2} \mathrm{PhB}(\mathrm{OH})_{2}+2,3$-dihydroxynaphthalene } & $k_{1}=$ & $(3.59 \pm 0.07) \times 10^{3}$ & $\mathrm{M}^{-1} \mathrm{~s}^{-1}$ \\
\hline & $k_{2} K_{\mathrm{a}}^{\mathrm{L}}+k_{3} K_{\mathrm{a}}^{\mathrm{B}}=$ & $(5.95 \pm 0.07) \times 10^{-4}$ & $\mathrm{~s}^{-1}$ \\
\hline & $k_{-1}=$ & $-b$ & \\
\hline & $k_{-2}+k_{-3}=$ & $-b$ & \\
\hline
\end{tabular}

${ }^{a}$ The $k_{\mathrm{obs}}$ vs. $C_{\mathrm{B}}$ plot was analyzed by the following equation (eq 3 with negligible participation of the $k_{4}\left(k_{-4}\right)$ path (see text)):

$$
k_{\mathrm{obs}}=\left(k_{1}+k_{2} K_{\mathrm{a} 1}^{\mathrm{L}}\left[\mathrm{H}^{+}\right]^{-1}+k_{3} K_{\mathrm{a}}^{\mathrm{B}}\left[\mathrm{H}^{+}\right]^{-1}\right) \mathrm{K}^{*-1} C_{\mathrm{B}}+k_{-1}\left[\mathrm{H}^{+}\right]+k_{-2}+k_{-3}
$$

${ }^{b}$ Not determined (negligible reaction path). 
Table S2a. The conditional rate constants $\left(k_{\text {obs }}\right)$ for the reaction of 2,4-difluorophenylboronic acid $\left(2,4-\mathrm{F}_{2} \mathrm{PhB}(\mathrm{OH})_{2}\right)$ with $2,2^{\prime}$-biphenol at $25.0^{\circ} \mathrm{C}$ and $I=0.10 \mathrm{M}{ }^{a}$

\begin{tabular}{|c|c|c|}
\hline $10^{8}\left[\mathrm{H}^{+}\right] / \mathrm{M}$ & $10^{3} C_{\mathrm{B}} / \mathrm{M}$ & $k_{\mathrm{obs}} / \mathrm{s}^{-1}$ \\
\hline \multirow[t]{4}{*}{6.76} & 2.04 & $\begin{array}{l}\text { 1.51, 1.59, 1.75, } 1.65,1.73,1.48,1.41,1.75,1.59,1.41,1.63,1.77,1.60 \text {, } \\
1.51,1.77,1.61,1.81\end{array}$ \\
\hline & 3.06 & $\begin{array}{l}\text { 1.96, 1.95, 1.86, 2.16, } 1.98,2.02,1.90,1.95,2.03,2.00,2.16,1.93,1.89 \text {, } \\
2.43,1.98,2.37\end{array}$ \\
\hline & 4.07 & $\begin{array}{l}\text { 2.04, 2.52, 2.45, 2.35, 2.60, 2.45, 2.44, 2.54, 2.25, 2.72, 2.51, 2.58, 2.20, } \\
\text { 2.59, } 2.73\end{array}$ \\
\hline & 5.09 & $\begin{array}{l}\text { 2.95, 3.02, 3.19, 3.11, 3.02, 2.90, 2.94, 2.43, 2.85, 3.33, 2.91, 2.90, 3.39, } \\
2.82,2.56,2.94\end{array}$ \\
\hline \multirow[t]{4}{*}{4.47} & 2.02 & $\begin{array}{l}\text { 1.09, } 1.28,1.26,1.20,1.14,1.15,1.09,1.24,1.21,1.23,1.16,1.12,1.06 \text {, } \\
1.18,1.20,1.16\end{array}$ \\
\hline & 3.04 & $\begin{array}{l}\text { 1.48, } 1.41,1.49,1.49,1.41,1.40,1.42,1.39,1.41,1.40,1.53,1.42,1.46 \text {, } \\
1.40,1.38\end{array}$ \\
\hline & 4.05 & $\begin{array}{l}\text { 1.82, } 1.78,1.84,1.82,1.74,1.90,1.94,1.75,1.88,1.92,1.72,1.81,1.81 \text {, } \\
1.90,1.86,1.85,1.75,1.88,1.81,1.86\end{array}$ \\
\hline & 5.06 & $2.11,2.19,2.23,2.15,2.05,2.44,2.39,2.12,2.06,2.14,2.16,2.17,2.10$ \\
\hline \multirow[t]{4}{*}{2.88} & 2.05 & $\begin{array}{l}\begin{array}{l}0.753,0.707,0.845,0.839,0.792,0.771,0.771,0.736,0.814,0.750 \text {, } \\
0.760,0.741\end{array}\end{array}$ \\
\hline & 3.08 & $\begin{array}{l}0.931,0.900,0.962,1.01,0.931,1.01,1.00,0.925,0.972,1.04,0.965 \text {, } \\
0.997,0.954,0.902,0.997,0.913,0.909,0.997\end{array}$ \\
\hline & 4.10 & $\begin{array}{l}\text { 1.13, } 1.12,1.12,1.10,1.08,1.19,1.23,1.19,1.16,1.10,1.14,1.18,1.18 \text {, } \\
1.15,1.15,1.14\end{array}$ \\
\hline & 5.13 & $1.39,1.55,1.57,1.52,1.34,1.51,1.55,1.45,1.42,1.44,1.33,1.38$ \\
\hline \multirow[t]{4}{*}{1.91} & 2.03 & $\begin{array}{l}0.485,0.504,0.499,0.510,0.510,0.514,0.516,0.510,0.516,0.518 \text {, } \\
0.516,0.526,0.513,0.494,0.512,0.492\end{array}$ \\
\hline & 3.05 & $\begin{array}{l}0.652,0.641,0.628,0.652,0.638,0.667,0.640,0.665,0.668,0.687 \text {, } \\
0.657,0.663,0.649,0.659,0.653,0.654,0.663\end{array}$ \\
\hline & 4.06 & $\begin{array}{l}0.768,0.795,0.792,0.773,0.801,0.782,0.786,0.825,0.819,0.782 \text {, } \\
0.792,0.822,0.791,0.800,0.804,0.798,0.798,0.797\end{array}$ \\
\hline & 5.08 & $\begin{array}{l}1.02,0.987,0.958,1.00,1.02,0.982,0.992,1.02,0.978,1.01,0.964 \text {, } \\
0.985,0.992,0.982,1.01,1.01,1.01\end{array}$ \\
\hline
\end{tabular}

${ }^{a} C_{\mathrm{L}}=(0.9-1.0) \times 10^{-5} \mathrm{M}$. 
Table S2b. The conditional rate constants $\left(k_{\mathrm{obs}}\right)$ for the reaction of 3-trifluoromethylphenylboronic acid $\left(3-\mathrm{CF}_{3} \mathrm{PhB}(\mathrm{OH})_{2}\right)$ with $2,2^{\prime}$-biphenol at $25.0^{\circ} \mathrm{C}$ and $I=0.10 \mathrm{M}^{a}$

\begin{tabular}{|c|c|c|}
\hline $10^{8}\left[\mathrm{H}^{+}\right] / \mathrm{M}$ & $10^{3} C_{\mathrm{B}} / \mathrm{M}$ & $k_{\mathrm{obs}} / \mathrm{s}^{-1}$ \\
\hline \multirow[t]{4}{*}{1.82} & 2.02 & 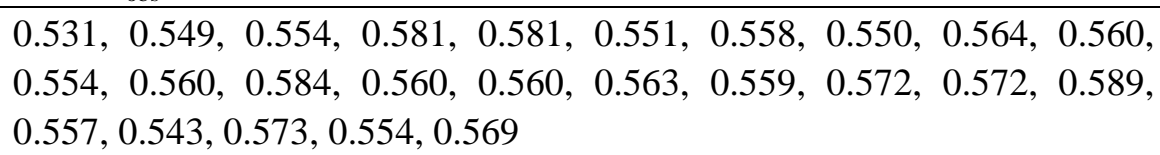 \\
\hline & 3.04 & 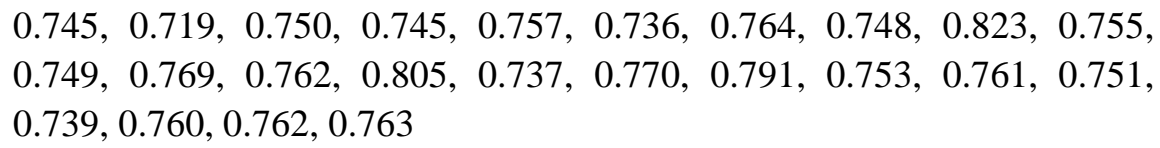 \\
\hline & 4.05 & $\begin{array}{l}0.947,0.968,0.943,0.983,0.965,0.933,0.964,0.956,0.993,0.979 \text {, } \\
0.975,0.974,0.963,0.968,0.947,0.989,0.940,0.969,0.952,1.01,0.976 \text {, } \\
0.975,0.990,0.947,0.964\end{array}$ \\
\hline & 5.06 & $\begin{array}{l}\text { 1.26, } 1.21,1.18,1.19,1.24,1.20,1.16,1.22,1.21,1.19,1.25,1.20,1.23 \text {, } \\
1.19,1.19,1.17,1.21,1.22,1.18,1.20,1.20\end{array}$ \\
\hline \multirow[t]{4}{*}{1.26} & 2.02 & $\begin{array}{l}\begin{array}{l}0.416,0.407,0.403,0.406,0.413,0.416,0.410,0.413,0.419,0.411 \text {, } \\
0.411,0.417,0.420,0.427,0.417\end{array}\end{array}$ \\
\hline & 3.03 & 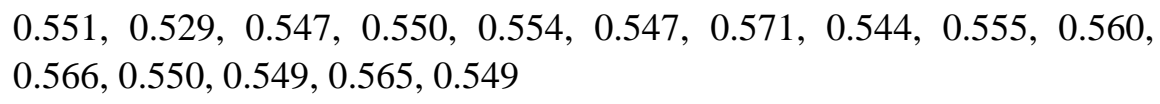 \\
\hline & 4.04 & $\begin{array}{l}\begin{array}{l}0.707,0.721,0.725,0.694,0.692,0.727,0.713,0.708,0.694,0.703 \text {, } \\
0.704,0.705,0.703,0.735,0.694\end{array}\end{array}$ \\
\hline & 5.05 & $\begin{array}{l}\begin{array}{l}0.892,0.887,0.879,0.886,0.882,0.900,0.893,0.891,0.888,0.874 \text {, } \\
0.878,0.889,0.880,0.881,0.886\end{array}\end{array}$ \\
\hline \multirow[t]{4}{*}{1.17} & 2.02 & $\begin{array}{l}0.402,0.410,0.403,0.418,0.405,0.401,0.409,0.412,0.436,0.413 \text {, } \\
0.410,0.401,0.424,0.409,0.405,0.417,0.411,0.415,0.410\end{array}$ \\
\hline & 3.03 & $\begin{array}{l}0.582,0.571,0.539,0.568,0.553,0.567,0.561,0.552,0.553,0.563 \text {, } \\
0.559,0.567,0.577,0.555,0.566,0.587,0.564,0.565,0.574,0.548,0.547\end{array}$ \\
\hline & 4.03 & $\begin{array}{l}0.737,0.691,0.708,0.718,0.737,0.728,0.706,0.737,0.707,0.742, \\
0.719,0.715,0.713,0.733,0.709,0.722,0.708,0.732,0.724,0.721,0.696\end{array}$ \\
\hline & 5.04 & 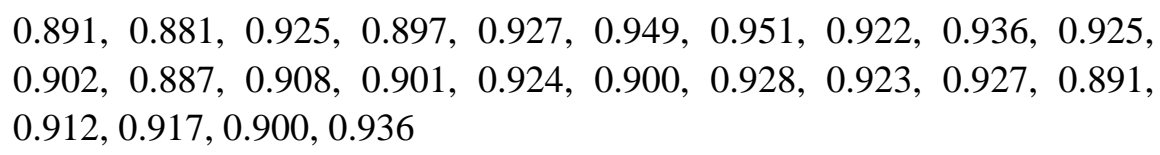 \\
\hline \multirow[t]{4}{*}{0.891} & 2.01 & $\begin{array}{l}0.330,0.344,0.349,0.348,0.347,0.340,0.357,0.349,0.350,0.341 \text {, } \\
0.352,0.346,0.352,0.342,0.353,0.347,0.341\end{array}$ \\
\hline & 3.02 & $\begin{array}{l}\begin{array}{l}0.466,0.471,0.462,0.459,0.456,0.453,0.467,0.454,0.454,0.456 \text {, } \\
0.468,0.457,0.457,0.463,0.460\end{array}\end{array}$ \\
\hline & 4.03 & $\begin{array}{l}0.582,0.599,0.585,0.591,0.579,0.579,0.564,0.583,0.594,0.586 \text {, } \\
0.589,0.604,0.587,0.575,0.580\end{array}$ \\
\hline & 5.04 & 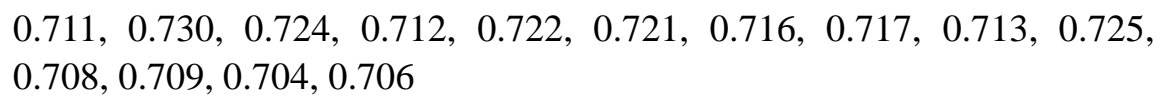 \\
\hline \multirow[t]{4}{*}{0.525} & 2.01 & $\begin{array}{l}\begin{array}{l}0.218,0.223,0.226,0.220,0.224,0.223,0.219,0.225,0.227,0.224, \\
0.220,0.229,0.226,0.230,0.222,0.227,0.221,0.226\end{array}\end{array}$ \\
\hline & 3.02 & $\begin{array}{l}0.292,0.275,0.284,0.276,0.280,0.294,0.299,0.287,0.284,0.293 \text {, } \\
0.292,0.283,0.287,0.291,0.291,0.287,0.287,0.272,0.288,0.284,0.280\end{array}$ \\
\hline & 4.03 & $\begin{array}{l}0.328,0.350,0.341,0.345,0.339,0.350,0.358,0.343,0.355,0.346 \text {, } \\
0.353,0.354,0.353,0.338,0.356,0.348,0.349,0.346,0.345,0.355,0.355\end{array}$ \\
\hline & 5.04 & $\begin{array}{l}0.419,0.421,0.409,0.411,0.423,0.411,0.417,0.412,0.409,0.414 \text {, } \\
0.414,0.416,0.424,0.418,0.413,0.420,0.421,0.427,0.416\end{array}$ \\
\hline
\end{tabular}

${ }^{a} C_{\mathrm{L}}=(1.0-1.1) \times 10^{-5} \mathrm{M}$. 
Table S2c. The conditional rate constants $\left(k_{\mathrm{obs}}\right)$ for the reaction of phenylboronic acid $\left(\mathrm{PhB}(\mathrm{OH})_{2}\right)$ with 2,2'-biphenol at $25.0^{\circ} \mathrm{C}$ and $I=0.10 \mathrm{M}^{a}{ }^{a}$

\begin{tabular}{|c|c|c|}
\hline $10^{9}\left[\mathrm{H}^{+}\right] / \mathrm{M}$ & $10^{3} C_{\mathrm{B}} / \mathrm{M}$ & $k_{\mathrm{obs}} / \mathrm{s}^{-1}$ \\
\hline \multirow[t]{4}{*}{4.17} & 4.00 & $\begin{array}{l}0.458,0.449,0.436,0.468,0.462,0.468,0.455,0.459,0.456,0.454 \text {, } \\
0.461,0.460,0.456,0.449,0.460,0.443\end{array}$ \\
\hline & 6.00 & $\begin{array}{l}0.544,0.574,0.555,0.581,0.562,0.561,0.552,0.561,0.557,0.561 \text {, } \\
0.573,0.570,0.557,0.545\end{array}$ \\
\hline & 8.01 & $\begin{array}{l}0.658,0.646,0.647,0.656,0.635,0.666,0.668,0.668,0.664,0.663 \text {, } \\
0.687,0.641,0.659\end{array}$ \\
\hline & 10.0 & $\begin{array}{l}0.779,0.773,0.789,0.749,0.732,0.750,0.756,0.762,0.788,0.751 \text {, } \\
0.756,0.745,0.795,0.738,0.755\end{array}$ \\
\hline \multirow[t]{4}{*}{2.24} & 4.01 & $\begin{array}{l}0.406,0.399,0.388,0.400,0.400,0.410,0.400,0.391,0.397,0.402 \text {, } \\
0.403,0.405\end{array}$ \\
\hline & 6.02 & $\begin{array}{l}0.472,0.488,0.493,0.475,0.453,0.470,0.480,0.462,0.487,0.491 \text {, } \\
0.486,0.470,0.467,0.478\end{array}$ \\
\hline & 8.02 & $\begin{array}{l}0.558,0.546,0.556,0.514,0.557,0.555,0.562,0.565,0.545,0.553 \text {, } \\
0.555,0.556,0.557\end{array}$ \\
\hline & 10.0 & $\begin{array}{l}0.603,0.618,0.671,0.597,0.656,0.572,0.600,0.621,0.613,0.584 \text {, } \\
0.614,0.612,0.587,0.620,0.670,0.650\end{array}$ \\
\hline \multirow[t]{4}{*}{1.55} & 4.01 & $0.373,0.386,0.376,0.373,0.379,0.380,0.376,0.376,0.376,0.388$ \\
\hline & 6.01 & $\begin{array}{l}0.457,0.449,0.426,0.436,0.439,0.447,0.423,0.448,0.429,0.432 \text {, } \\
0.445,0.441,0.442,0.448,0.451,0.427\end{array}$ \\
\hline & 8.02 & $\begin{array}{l}0.490,0.511,0.512,0.502,0.514,0.509,0.519,0.496,0.515,0.501 \text {, } \\
0.504,0.498,0.500,0.500\end{array}$ \\
\hline & 10.0 & $\begin{array}{l}0.561,0.578,0.570,0.565,0.573,0.566,0.563,0.572,0.578,0.585 \text {, } \\
0.588,0.576,0.556,0.567,0.575\end{array}$ \\
\hline \multirow[t]{4}{*}{1.12} & 4.02 & $\begin{array}{l}0.348,0.349,0.352,0.341,0.349,0.363,0.352,0.351,0.352,0.356 \text {, } \\
0.356,0.357,0.356,0.348,0.346,0.350\end{array}$ \\
\hline & 6.02 & $\begin{array}{l}0.380,0.391,0.396,0.388,0.405,0.410,0.400,0.400,0.399,0.409 \text {, } \\
0.387,0.412\end{array}$ \\
\hline & 8.03 & $\begin{array}{l}0.438,0.445,0.443,0.464,0.475,0.453,0.451,0.455,0.439,0.456 \text {, } \\
0.441,0.458\end{array}$ \\
\hline & 10.0 & $\begin{array}{l}0.500,0.488,0.509,0.497,0.478,0.515,0.491,0.523,0.497,0.503 \text {, } \\
0.511,0.517,0.517,0.486,0.489,0.520\end{array}$ \\
\hline
\end{tabular}

${ }^{a} C_{\mathrm{L}}=(1.0-1.1) \times 10^{-5} \mathrm{M}$. 
Table S3. The conditional rate constants $\left(k_{\mathrm{obs}}\right)$ for the reaction of 2,4-difluorophenylboronic acid $\left(2,4-\mathrm{F}_{2} \mathrm{PhB}(\mathrm{OH})_{2}\right)$ with 2,3-dihydroxynaphthalene at $25.0^{\circ} \mathrm{C}$ and $I=0.10 \mathrm{M} .^{a}$

\begin{tabular}{|c|c|c|}
\hline $10^{7}\left[\mathrm{H}^{+}\right] / \mathrm{M}$ & $10^{3} C_{\mathrm{B}} / \mathrm{M}$ & $k_{\mathrm{obs}} / \mathrm{s}^{-1}$ \\
\hline \multirow[t]{4}{*}{3.02} & 2.00 & $\begin{array}{l}\text { 9.88, 8.83, 9.03, 9.27, 8.89, 8.54, 8.60, 8.86, 8.32, 8.51, 8.86, 9.58, 8.74, } \\
\text { 8.61, 8.76, 9.37, 8.28, 8.83, 8.65, 9.18, 8.62, 8.71, 8.48, 8.53, 8.61 }\end{array}$ \\
\hline & 3.00 & $\begin{array}{l}12.1,12.6,12.9,12.4,12.3,13.6,12.9,13.1,12.2,13.0,13.2,14.4,13.2, \\
12.5,14.0,13.8,11.9,13.7,12.9,12.6,13.4,13.0,13.6,12.0,12.7\end{array}$ \\
\hline & 4.01 & $\begin{array}{l}\text { 17.0, 18.1, } 18.9,17.4,18.3,15.0,16.6,14.7,16.8,17.8,18.1,16.6,16.4 \text {, } \\
18.3,20.5,17.1,20.0,15.5,16.7,16.0,19.0,18.8,18.4,18.4\end{array}$ \\
\hline & 5.01 & $\begin{array}{l}22.9,27.7,21.9,24.7,23.3,20.5,23.2,21.8,22.2,24.4,22.5,18.5,23.5 \text {, } \\
20.5,20.1,20.5,22.1,23.4,20.1\end{array}$ \\
\hline \multirow[t]{4}{*}{1.48} & 2.00 & $\begin{array}{l}10.0,9.53,10.1,10.2,10.2,9.13,9.65,9.75,9.59,9.94,9.85,9.25,10.5 \text {, } \\
9.71,10.1,9.20,9.80,10.0,9.79,10.3,9.99,9.52,10.2,9.15,9.14\end{array}$ \\
\hline & 3.01 & $\begin{array}{l}\text { 13.2, } 13.5,15.3,14.7,14.4,15.8,13.6,14.9,15.3,14.9,14.4,14.1,14.0 \text {, } \\
13.9,15.4,15.4,16.1,13.7,14.4,14.2,14.9,14.2,13.7,15.5,13.2\end{array}$ \\
\hline & 4.01 & $\begin{array}{l}\text { 19.5, 20.4, 20.7, 22.8, 20.5, 22.2, 19.4, 19.3, 20.1, 23.6, 22.5, 20.1, 20.3, } \\
19.8,20.7,19.4,19.4,19.5,20.4,18.8,19.0,20.4\end{array}$ \\
\hline & 5.01 & $\begin{array}{l}25.3,21.9,23.7,21.9,23.8,28.0,26.3,22.5,25.7,24.0,22.5,27.0,22.9 \text {, } \\
27.7,25.2,26.8,29.1,23.7,26.9,22.6,23.6,23.3,27.7,23.4,25.2\end{array}$ \\
\hline \multirow[t]{4}{*}{0.759} & 2.00 & $\begin{array}{l}11.2,10.5,10.2,11.1,11.5,10.4,10.8,10.4,11.1,11.1,10.4,9.92,11.2 \text {, } \\
10.5,11.4,10.9,10.8,10.1,11.2,11.6,11.2,11.1,10.9,10.6,10.5\end{array}$ \\
\hline & 3.00 & $\begin{array}{l}\text { 17.1, } 16.2,17.8,15.6,16.5,15.2,16.9,15.4,17.0,16.9,15.3,19.0,16.4, \\
17.1,17.1,14.8,16.6,16.8,17.9,15.5,17.3,17.4,17.2,15.9,16.3\end{array}$ \\
\hline & 4.00 & $\begin{array}{l}\text { 27.5, 23.5, 22.7, 25.3, 24.3, 22.7, 22.1, 25.4, 22.9, 25.7, 20.2, 22.2, 23.2, } \\
\text { 23.4, 21.7, 21.3, 21.6, 26.7, 22.3, 24.0, 24.0, } 22.3\end{array}$ \\
\hline & 5.00 & $\begin{array}{l}\text { 32.4, 29.6, 29.6, 27.6, 27.3, 34.9, 28.5, 37.5, 31.1, 30.2, 27.1, 25.8, 30.3, } \\
24.0,28.2,27.1,26.1,29.1,28.5,31.1,25.5,21.8\end{array}$ \\
\hline \multirow[t]{4}{*}{0.457} & 2.00 & $\begin{array}{l}11.8,11.3,11.7,12.1,12.3,11.7,11.8,11.5,11.9,11.9,12.9,12.0,11.9, \\
10.8,11.9,11.1,12.9,10.8,11.4,12.9,13.4,10.7,11.2,12.8\end{array}$ \\
\hline & 3.00 & $\begin{array}{l}\text { 18.6, 16.3, 18.2, 16.8, 16.9, 18.3, 17.7, 18.1, 16.9, 18.7, 16.9, 17.5, } 17.6 \text {, } \\
16.6,17.1,18.7,18.3,18.0,18.1,18.5,17.5,15.9,19.1,18.2,17.7\end{array}$ \\
\hline & 4.00 & 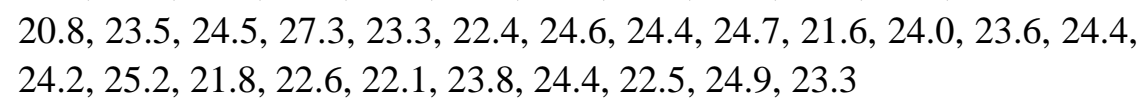 \\
\hline & 4.99 & $\begin{array}{l}\text { 31.0, 37.8, 29.8, 28.9, 28.4, 30.0, 30.1, 28.1, 30.1, 24.6, 32.7, 27.5, 31.8, } \\
\text { 32.2, 32.2, 33.3, 29.7, 28.8, 26.8, 30.8, 26.2 }\end{array}$ \\
\hline
\end{tabular}

\footnotetext{
${ }^{a} C_{\mathrm{L}}=1.0 \times 10^{-5} \mathrm{M}$.
} 


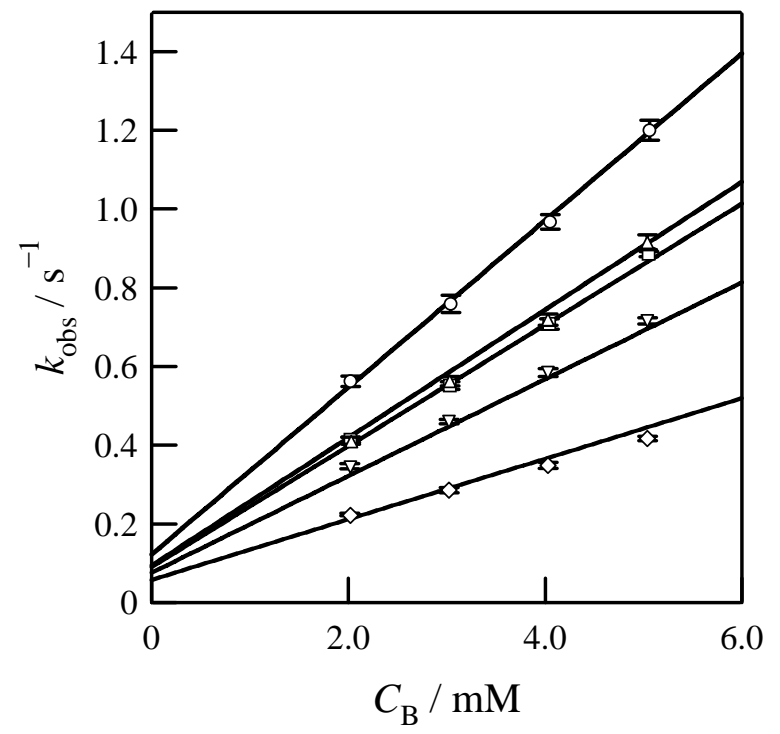

Figure S1. Dependence of $k_{\mathrm{obs}}$ on $C_{\mathrm{B}}$ for the reaction of 3- $\mathrm{CF}_{3} \mathrm{PhB}(\mathrm{OH})_{2}$ with 2,2'-biphenol $\left(1 \times 10^{-5} \mathrm{M}\right)$ in alkaline solution at $I=0.10 \mathrm{M}$ and $T=25^{\circ} \mathrm{C} . \quad\left[\mathrm{H}^{+}\right] / \mathrm{M}=1.82 \times 10^{-8}$ (circle), $1.26 \times 10^{-8}$ (square), $1.17 \times 10^{-8}$ (triangle), $8.91 \times 10^{-9}$ (wedge), $5.25 \times 10^{-9}$ (diamond). 


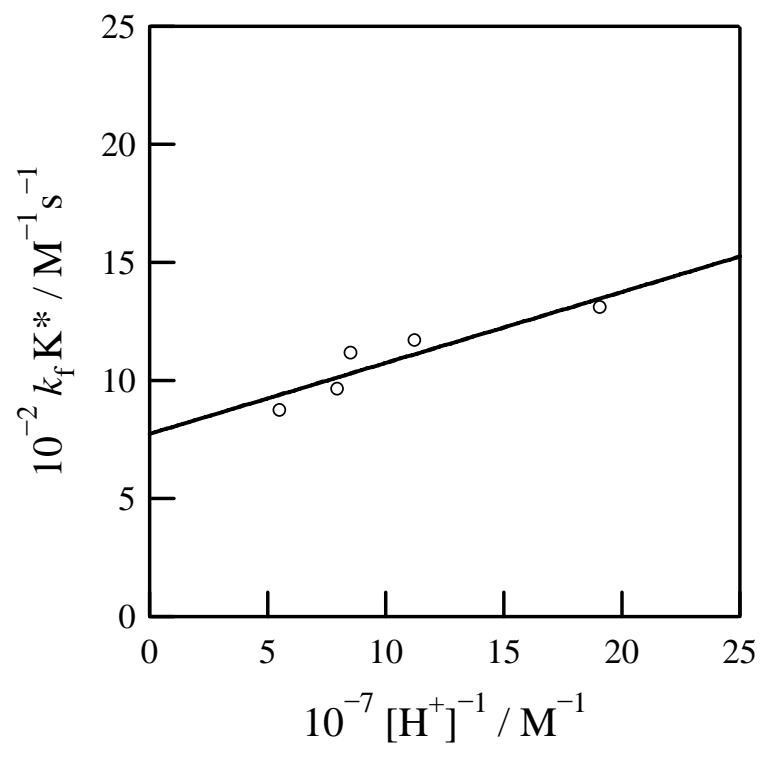

Figure S2. The plot of $k_{\mathrm{f}} \mathrm{K}^{*}$ against $\left[\mathrm{H}^{+}\right]^{-1}$ for the reaction of $3-\mathrm{CF}_{3} \mathrm{PhB}(\mathrm{OH})_{2}$ with 2,2'-biphenol at $I=0.10 \mathrm{M}$ and $T=25^{\circ} \mathrm{C}$. 


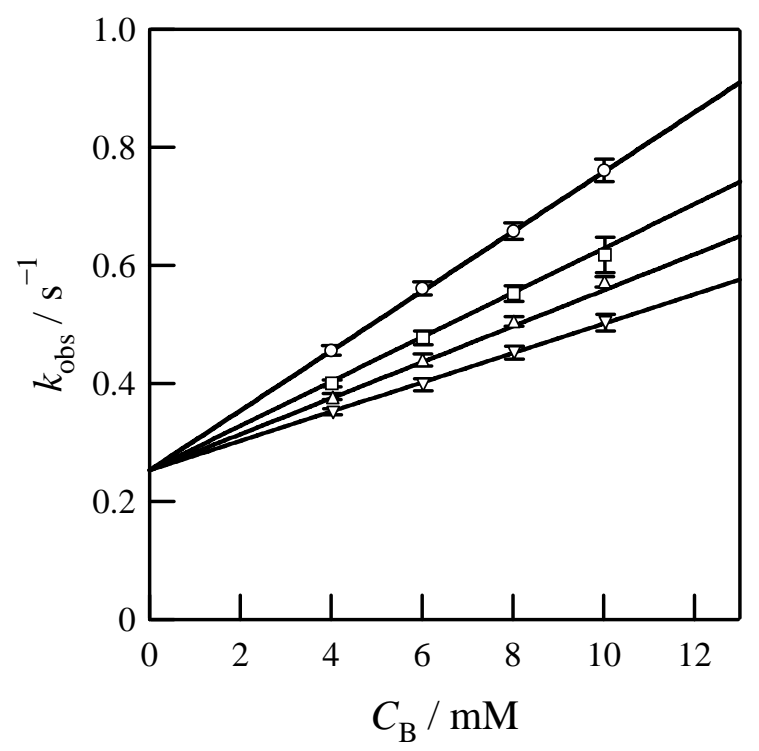

Figure S3. Dependence of $k_{\mathrm{obs}}$ on $C_{\mathrm{B}}$ for the reaction of $\mathrm{PhB}(\mathrm{OH})_{2}$ with 2,2'-biphenol (1 x $10^{-5} \mathrm{M}$ ) in alkaline solution at $I=0.10 \mathrm{M}$ and $T=25^{\circ} \mathrm{C} . \quad\left[\mathrm{H}^{+}\right] / \mathrm{M}=4.17 \times 10^{-9}$ (circle), 2.24 x $10^{-9}$ (square), $1.55 \times 10^{-9}$ (triangle), $1.12 \times 10^{-9}$ (wedge). 


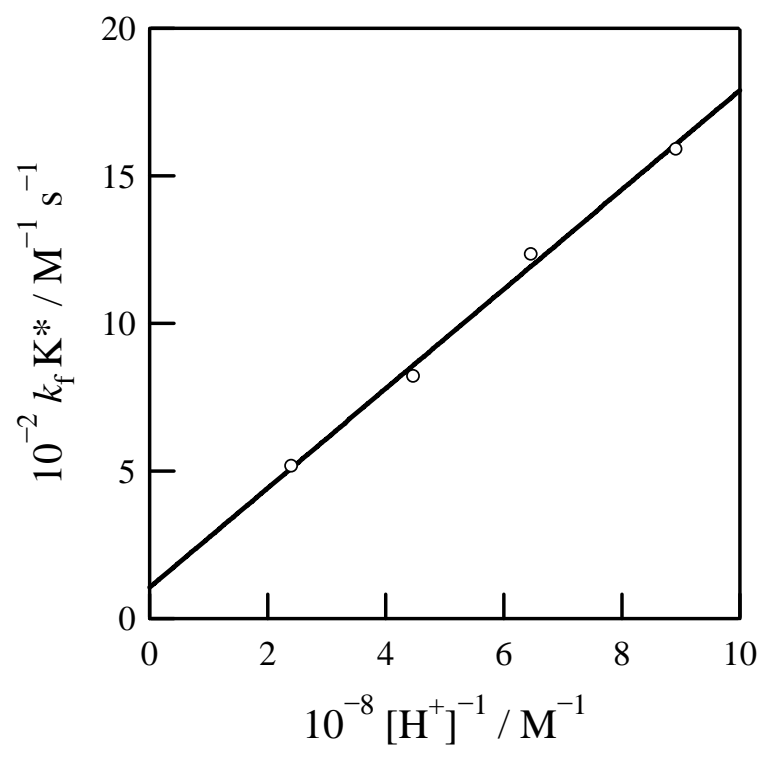

Figure S4. The plot of $k_{\mathrm{f}} \mathrm{K}^{*}$ against $\left[\mathrm{H}^{+}\right]^{-1}$ for the reaction of $\mathrm{PhB}(\mathrm{OH})_{2}$ with 2,2'-biphenol at $I=0.10 \mathrm{M}$ and $T=25^{\circ} \mathrm{C}$. 


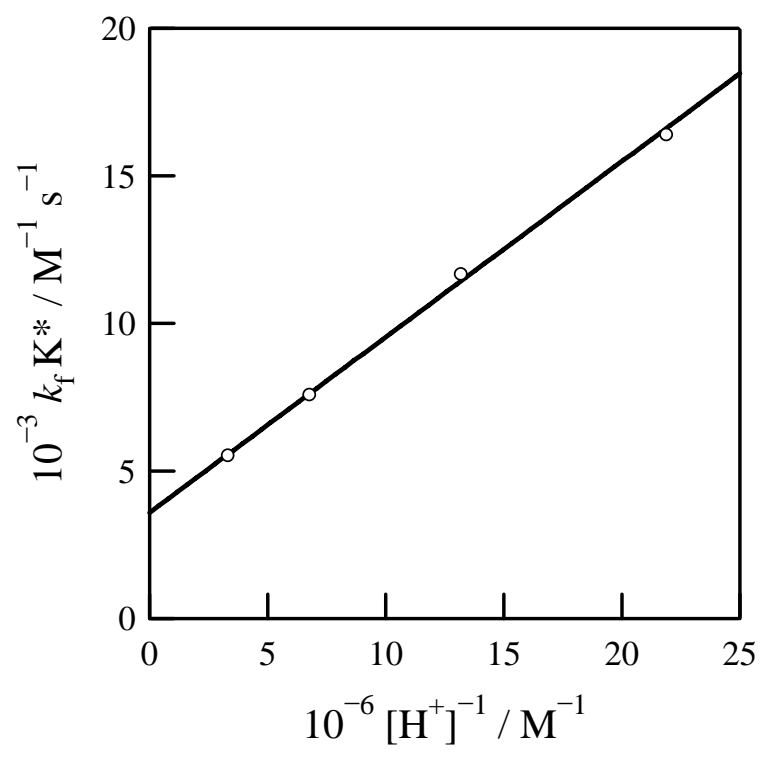

Figure S5. The plot of $k_{\mathrm{f}} \mathrm{K}^{*}$ against $\left[\mathrm{H}^{+}\right]^{-1}$ for the reaction of $2,4-\mathrm{F}_{2} \mathrm{PhB}(\mathrm{OH})_{2}$ with 2,3-dihydroxynaphthalene at $I=0.10 \mathrm{M}$ and $T=25^{\circ} \mathrm{C}$. 